ఠ

\title{
Eltrombopag - a novel approach for the treatment of chronic immune thrombocytopenic purpura: review and safety considerations
}

This article was published in the following Dove Press journal:

Drug Design, Development and Therapy

29 June 2010

Number of times this article has been viewed

\author{
Lucy Cook \\ Nichola Cooper \\ Department of Haematology, Imperial \\ Health Care NHS Trust, Hammersmith \\ Hospital, London, UK
}

\begin{abstract}
Eltrombopag is one of a number of novel agents recently developed for use in the treatment of patients with immune thrombocytopenia (ITP). Rather than preventing destruction of platelets, these agents increase the production of platelets, presumably overwhelming the immune system resulting in normal platelet counts in individuals refractory to or dependent on other therapies. These treatments are well tolerated and in randomized controlled trials show an improvement in platelet counts and a reduction in bleeding in refractory patients. This article summarizes the development of this new class of drug and evaluates the safety and efficacy of eltrombopag in patients with ITP.
\end{abstract}

Keywords: eltrombopag, immune thrombocytopenic purpura, thrombopoietin

\section{Introduction}

Immune thrombocytopenia (ITP) is an acquired disease of low platelet counts (less than $100 \times 10^{9} / \mathrm{L}$ ) with no clear initiating or underlying cause. ITP may manifest with bleeding symptoms which correlate, to some extent, with the severity of thrombocytopenia. The majority of cases are considered primary, where a precipitating cause is unknown, whereas others are attributed as secondary to coexisting medical conditions such as systemic lupus erythematosus, chronic lymphocytic leukemia, and immune deficiency disorders. Although the definition of chronic ITP has been debated, a recent consensus statement suggests this should be considered if platelets remain below normal for more than 12 months. ${ }^{1}$

The pathogenesis of ITP is complex but involves premature destruction of platelets mediated by anti-platelet antibodies ${ }^{2}$ and/or by direct $\mathrm{T}$ cell mediated platelet lysis. ${ }^{3} \mathrm{In}$ addition to increased platelet destruction, there is also evidence of decreased platelet production. In 1989, Gernsheimer et $\mathrm{al}^{4}$ described inappropriately normal or decreased platelet production in some patients with ITP. Further studies have shown increased apoptosis in megakaryocytes in patients with ITP ${ }^{5}$ and CD34+ cells grown with plasma from patients with ITP have impaired development of megakaryocytes and production of platelets suggesting that the anti-platelet antibody not only results in premature destruction of platelets but also impairs platelet production. ${ }^{2}$

Treatment aims in ITP have previously concentrated on preventing platelet destruction using steroids, immunoglobulins, immunosuppressive agents and splenectomy. ${ }^{6,7}$ However, many of these treatments are unsatisfactory with the morbidity and mortality of this disorder relating as much from the side effects of treatment such as infections as
Department of Haematology, Imper Health Care NHS Trust, Hammersmith Hospital, Du Cane Road, London WI2 OHS

Email n.cooper@imperial.ac.uk 
from bleeding. ${ }^{6}$ Novel treatment strategies that do not include immunosuppression are needed. Following the understanding of the role of megakaryocytes in the pathogenesis of ITP, and the appreciation of the low level of platelet production in patients with ITP, the potential use of agents with thrombopoietic ability in ITP has been explored.

This review will focus on the development and therapeutic use of one of these agents eltrombopag, in chronic ITP.

\section{Thrombopoiesis}

Over the last 25 years a number of hemopoietic growth factors with thrombopoietic activity have been identified including granulocyte-macrophage colony stimulating factor (GM-CSF); stem cell factor (c-kit ligand); interleukin 1 (IL-1), IL-3, IL-6 and IL-11; and thrombopoietin (TPO). ${ }^{8-11,12}$ Gene-targeting studies have demonstrated that TPO, the c-MPL ligand, is the most important physiologic regulator of steady-state megakaryocyte and platelet production. ${ }^{13,14}$

\section{TPO and its biology}

TPO is a large molecule (95 KDa) synthesized primarily in the liver as a single 333 -amino acid precursor protein. The receptor binding domain of TPO contains 153 amino-terminal residues: Each TPO molecule binds two TPO receptors, one via a high-affinity binding site (13 amino acids) and the other via a low affinity binding site (11 amino acids). ${ }^{15}$ Binding of TPO to its receptor triggers the activation of the cytoplasmic tyrosine kinases janus kinase (JAK)2 and subsequent signal transducers and activators (STAT)5, phosphoinositide-3 kinase and Ras-mitogen-activated protein kinase (MAPK). Activation of these pathways leads to changes in gene expression that promote progression along the megakaryocytic pathway, ultimately leading to the release of platelets into the peripheral circulation (Figure 1). ${ }^{16}$

The TPO receptor is found on megakaryocyte precursor cells, megakaryocytes, and platelets, as well as on stem cells and early bone marrow progenitor cells of all lineages.

\section{Physiologic control of TPO production}

There are no known physiologic sensors of the platelet count or mass and studies have suggested that hepatic transcription and translation of the TPO gene to be constant. ${ }^{17,18}$ TPO is cleared by avid TPO receptors present on platelets and possibly megakaryocytes. Significantly elevated endogenous TPO levels have been observed in disorders in which there is diminished platelet production and therefore reduced TPO clearance, although thrombopoietin levels are typically normal in patients with ITP. During times of normal or increased platelet mass there is increased clearance of TPO leading to normalization of TPO levels. ${ }^{19,20}$

\section{First-generation recombinant thrombopoietin}

Since TPO was cloned in 1994 several TPO molecules have been developed for clinical evaluation. The first generation recombinant TPOs, recombinant human thrombopoietin (rh TPO) and pegylated human recombinant megakaryocyte growth and development factor

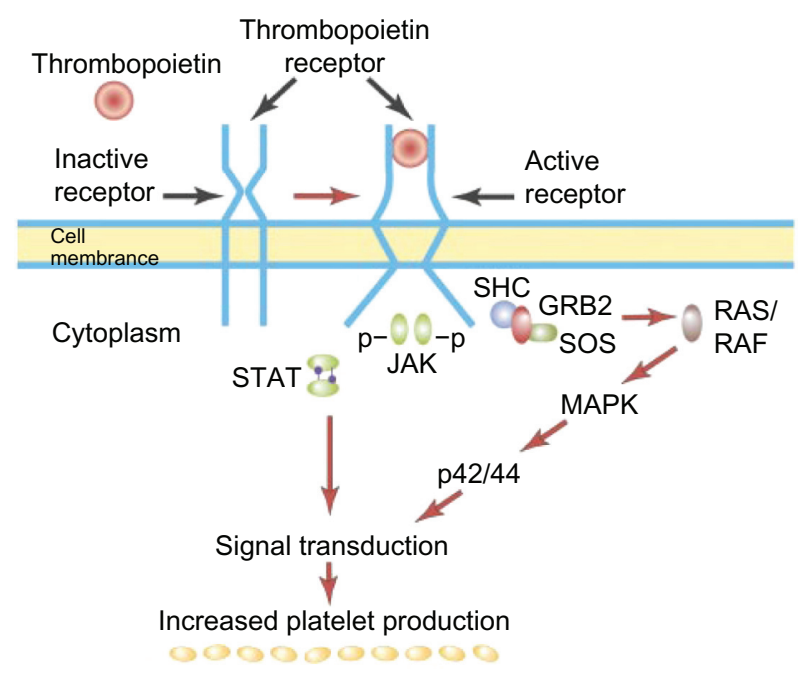

Figure I TPO binds the distal part of the inactive TPO (cMPL) receptor and creates an activated receptor that initiates many downstream signal transduction events (JAK STAT and mitogen activated protein kinase signal pathways) whereas eltrombopag interacts with the transmembrane domain of c-MPL. Reproduced with permission from Kuter DJ. New thrombopoietic growth factors. Blood. 2007; 109(I I): 4607-46I6. ${ }^{46}$ Copyright ( 2007 The American Society of Hematology. 
(PEG-rHuMGDF), developed in 1995 have undergone considerable preclinical and clinical studies. Platelet count response to single dose administration of both recombinant TPOs was demonstrated. A 5-day time lag occurred, presumably due to TPO stimulation of early and not late megakaryocyte precursors, with a peak platelet count at 12 to 14 days, a log-linear dose response curve and a return to baseline platelet count by day 28 with no rebound thrombocytopenia. ${ }^{21,22}$

Promising clinical trial results were seen in the areas of chemotherapy-induced thrombocytopenia, stem cell transplantation, myelodysplastic syndromes ${ }^{21,23-28}$ and prior to platelet apheresis. ${ }^{29}$ Recombinant TPO also showed efficacy in patients with immune thrombocytopenia (ITP); $;^{30}$ platelet counts rose dramatically in 3 of 4 patients who had received 7 daily treatments with PEG-rHuMGDF. Following on from these promising clinical trial results a large safety study of PEG-rHuMGDF was undertaken in healthy volunteers. ${ }^{31}$ Each volunteer was given a monthly dose of either PEG-rHuMGDF or placebo for up to 3 months. However, 13 of 535 subjects who received PEG-rHuMGDF developed an anti-TPO antibody compared with none of the 532 placebo-treated subjects. This antibody was later found to be an $\operatorname{IgG}_{4}$ subclass and was cross reactive with endogenous TPO neutralizing its activity and resulting in severe thrombocytopenia (platelet counts 10 to $20 \times 10^{9} / \mathrm{L}$ ). In 2 patients pancytopenia developed and some volunteers required treatment with ciclosporin and rituximab to eliminate the autoantibody and restore normal blood counts. All volunteers subsequently recovered. ${ }^{22,31}$ Subsequent analysis of 650 cancer chemotherapy patients treated with PEG-rHuMGDF identified at least four who also developed thrombocytopenia, believed to be due to auto-antibody formation. No subject treated with rhTPO developed neutralizing antibodies. However, clinical development of both recombinant TPOs was ultimately discontinued and many of the clinical studies undertaken between 1994 and 1999 remain unpublished. ${ }^{15}$

\section{Alternative TPO agonists}

Due to the promising platelet responses of the first-generation agents, there has been focus on the development of safe nonimmunogenic growth factors. These can be considered in 3 classes: TPO nonpeptide mimetics, TPO peptide mimetics and TPO antibody mimetics. Each class has a unique structure and therefore a unique mode of activation of the TPO receptor. This review article focuses on eltrombopag, the most extensively trialled drug of the TPO nonpeptide mimetic class.

\section{Eltrombopag (SB497 I I 5)}

Eltrombopag was first developed by Duffy et al following the screening of libraries of small molecules using an assay to identify structures that stimulate the TPO receptor-linked transduction pathways. ${ }^{32,33}$ The libraries of small compounds underwent further biological modification prior to entering clinical trials with the sulfonate group being replaced by a biphenyl carboxylate compound in order to yield higher oral bioavailability. ${ }^{34}$

This class of molecule has several important characteristics: Firstly it is extremely small (564.6 Da) and binds very precisely to the TPO receptor. Secondly it is only able to do this in humans and non-human primates,${ }^{34}$ preventing efficacy studies in other animal models. The species specificity is due to the amino acid present at position 499 in the TPO receptor transmembrane region (histidine in primates and leucine in all other species). Thirdly eltrombopag appears to activate the TPO receptor by binding to the transmembrane domain unlike TPO (and TPO peptide mimetics) which bind to the distal homology receptor domain.

Eltrombopag has high potency in in vitro assays for activation of the STAT and MAPK signaling pathways. It activates TPO receptor with kinetics similar to rhTPO resulting in proliferation of TPO-dependent cell lines and induces the differentiation of bone marrow precursor cells. These activities of eltrombopag have been shown to be dependent on the expression of TPO receptor. Because eltrombopag does not bind to the same site on TPO receptor as TPO this prevents competitive binding and allows eltrombopag and TPO to have additive cell-signaling effects. ${ }^{34}$

\section{Clinical studies with eltrombopag}

Phase 1 clinical trials of an oral once daily capsule given to 73 healthy volunteers were first reported in $2007 .{ }^{35} \mathrm{~A}$ dosedependent increase in platelet count at doses of $30 \mathrm{mg}$ or more given daily for 10 days was reported, with no adverse events. Furthermore, there was no evidence of rebound thrombocytopenia following discontinuation of treatment. Platelet function, as measured by platelet aggregation and activation, was not affected by the administration of eltrombopag. ${ }^{35}$

Following the success of these phase I studies, Eltrombopag is being studied at phase I/II and phase III in ITP, chemotherapy-induced thrombocytopenia, myelodysplastic syndrome (MDS) and hepatitis C infection.

\section{Chronic ITP}

A phase I/II study of 118 patients (in 44 centers) with refractory or relapsed chronic ITP was reported in 2007. This study 
enrolled patients in a randomized, double blind, placebocontrolled study to receive either placebo or eltrombopag at 30-mg, 50-mg, or 75-mg doses (in a 1:1:1:1 ratio) for 6 weeks. The primary endpoint was a platelet count above $50 \times 10^{9} / \mathrm{L}$ on day 43. Randomization was stratified for concomitant steroid use, previous splenectomy and baseline platelet count. Eltrombopag was well tolerated and achieved dose related responses; the percentage of patients with a platelet count above $50 \times 10^{9} / \mathrm{L}$ on day 43 were $11 \%, 28 \%, 70 \%$ and $81 \%$ for the placebo, $30 \mathrm{mg}, 50 \mathrm{mg}$ and $75 \mathrm{mg}$ doses respectively. The median platelet counts on day 43 for the groups receiving 30,50 , and $75 \mathrm{mg}$ of eltrombopag were $26 \times 10^{9} / \mathrm{L}, 128 \times$ $10^{9} / \mathrm{L}$, and $183 \times 10^{9} / \mathrm{L}$, respectively; for the placebo group the median platelet count was $16 \times 10^{9} / \mathrm{L}$.

Significantly, this trial demonstrated a decreased bleeding tendency in those patients treated with higher doses of eltrombopag; by day 15 over $80 \%$ of patients on higher doses (50 mg and $75 \mathrm{mg}$ ) had an increased platelet count with significantly less bleeding: During the 6-week trial, the incidence of bleeding was $14 \%, 17 \%, 7 \%$ and $4 \%$ for the placebo, 30-mg, 50-mg and 75-mg doses respectively. The counts were maintained for the 6 -week trial period but returned to baseline on stopping treatment in the majority of patients. In this study there was one reported death in a patient with multiple co-morbidities, not felt to be related to the use of eltrombopag. Eltrombopag was associated with minor adverse events (eg, headaches in 20\%) and was well tolerated. There were no other definite treatment-related adverse events reported during the study. The incidence and severity of adverse events were similar for all four study groups.

In a following phase II study, patients enrolled in the initial 6-week study were eligible to enter a long-term, open-label extension study - EXTEND study (Eltrombopag Extended Dosing Study). In this study, the drug was administered orally daily aiming for a target platelet count of 50 to $200 \times 10^{9} / \mathrm{L}$, and with the intention that concurrent ITP medications (eg, corticosteroids) could be tapered. Interim analysis of 89 evaluable subjects with a treatment duration of 151 days showed that of the 61 subjects $(73 \%)$ with a baseline platelet count below $30 \times 10^{9} / \mathrm{L}, 43$ achieved a platelet count of $>50 \times 10^{9} / \mathrm{L}$. Most were able to maintain this platelet count elevation and showed a trend toward fewer bleeding events. ${ }^{36}$

Similar promising responses were reported in a multinational phase III randomized, placebo-controlled study of 110 patients with chronic ITP. ${ }^{37}$ The purpose of this study was to evaluate the efficacy, safety and tolerability of once daily eltrombopag (50 mg) and to explore the efficacy of a dose escalation to $75 \mathrm{mg}$. Patients with platelet counts less than
$30 \times 10^{9} / \mathrm{L}$ were randomized to either placebo (37 patients) or oral eltrombopag $50 \mathrm{mg}$ once daily (73 patients) in a 1:2 ratio. Dose escalation to $75 \mathrm{mg}$ once daily was allowed if the platelet count was less than $50 \times 10^{9} / 1$ at 3 weeks (33 of 74 patients). The primary endpoint was the number of patients with a platelet count $>50 \times 10^{9} / \mathrm{L}$ at day 43 . At 6 weeks, the primary endpoint had been reached in $6(16 \%)$ placebo and 43 (59\%) eltrombopag treated patients. Response was not affected by predefined study stratification variables (baseline platelet count, previous splenectomy, concomitant ITP drugs) or by the number of previous treatments. In addition the bleeding incidence was significantly lower in the eltrombopag group with WHO grade 2-4 bleeding observed in $16 \%$ of patients compared with $36 \%$ of placebo patients (odds ratio 0.49 [95\% confidence interval 0.26 to 0.89 ] $P=0.021)$. Again adverse events were infrequent with no significant differences between the two groups. Slight increases of liver enzymes were noted in 6 patients in the eltrombopag group. Cataracts were closely monitored during the study due to concerns raised in preclinical rodent studies but no excess risk has been identified in any trial. ${ }^{37}$

Antiplatelet antibodies and thrombopoietin levels were not assessed in these patients and it is unclear what effect these variables will have on response to treatment. It has, however, been suggested that thrombopoietic agents such as eltrombopag may result in improved immune regulation by regulatory $\mathrm{T}$ cells and these agents may restore immune tolerance. $^{38}$

\section{Quality of life}

The impact of eltrombopag on quality of life in chronic ITP was evaluated in both the phase II and III study with no significant difference found between placebo and treatment arms and between responders and non-responders over the 6-week time period. ${ }^{37}$ This is possibly due to the short follow-up and small sample size for this secondary endpoint.

Health related questionnaires are now incorporated into a number of the more recent clinical trials. In a survey completed by 1542 respondents with chronic ITP, patients were willing to accept significant risks of thromboembolic events, liver abnormalities, and rebound thrombocytopenia for greater efficacy benefit, as well as to avoid the use of corticosteroids. ${ }^{39}$

\section{Hepatitis C related thrombocytopenia}

McHutchison and colleagues reported in 2007 the results of a multicenter, randomized, blinded, placebo-controlled phase II trial of eltrombopag versus placebo in 74 patients with chronic hepatitis $\mathrm{C}$ infection who had compensated liver disease with a 
platelet count 20 to $70 \times 10^{9} / \mathrm{L}$. These patients were randomized to eltrombopag ( $30 \mathrm{mg}, 50 \mathrm{mg}$, or $75 \mathrm{mg}$ daily) or placebo daily for 4 weeks. All patients who completed 4 weeks of treatment and reached a platelet count of $\geq 70 \times 10^{9} / \mathrm{L}$ received antiviral therapy (pegylated interferon with ribavarin) concomitant with eltrombopag for 8 to 12 weeks.

The results showed that none of the patients in the placebo arm reached the primary end point; however 20 of 23 patients receiving $75 \mathrm{mg}$ eltrombopag attained a platelet count $\geq 100 \times$ $10 \% / \mathrm{L}$. Compared with only $6 \%$ of the placebo group, 15 of 23 patients (or 65\%) in the high-dose eltrombopag group were able to complete 12 weeks of antiviral treatment without dose interruptions because they had platelets $\geq 50 \times 10^{9} / \mathrm{L}$.

The most frequent adverse event in the 4 week eltrombopag phase was headache, reported by 3 patients on placebo, and 5,3 , and 4 patients on eltrombopag $30 \mathrm{mg}, 50 \mathrm{mg}$, and $75 \mathrm{mg}$, respectively. There are no safety concerns to date, although the combined numbers of patients treated are, to date, only in the hundreds. Phase III clinical trials evaluating eltrombopag in hepatitis $\mathrm{C}$ infection are in progress. Together they have a target enrolment of over one thousand patients. ${ }^{40}$

\section{Potential serious adverse effects}

Eltrombopag appears to have very good short-term tolerability with no serious adverse events reported. However, this drug has only been used in hundreds of patients and the maximum duration of treatment with eltrombopag reported is 151 days. ${ }^{15}$ Potential adverse effects include:

\section{Antibody formation}

There have been no reports of antibody formation (there is no sequence homology with TPO).

\section{Thrombosis or thrombocytosis}

It has been suggested that ITP (and/or its treatment) creates a prothrombotic state, possibly due to the large number of new (and active) platelets being formed. In the placebo-controlled ITP studies there has been no difference reported in thrombotic events between treatment or placebo arms. ${ }^{15} \mathrm{~A}$ comparative analyses of eltrombopag and TPO on in vitro platelet function demonstrated that eltrombopag stimulates platelet signal transduction with little or no effect on overall platelet function, in contrast to TPO, which significantly primes platelet activation. ${ }^{41}$

\section{Rebound thrombocytopenia}

In the phase III trial of chronic ITP in over 100 patients, platelet counts remained above $50 \times 10^{9} / \mathrm{L}$ in approximately half the patients for a week following discontinuation. They generally returned to baseline levels within 2 weeks of discontinuing therapy. In 2 patients recurrence of bleeding symptoms occurred with a decrease in platelet counts to $<10 \times 10^{9} / \mathrm{L}$ and at least $10 \times 10^{9} / \mathrm{L}$ lower than baseline value. ${ }^{37}$

\section{Reticulin fibrosis}

Bone marrow (BM) reticulin (without collagen fibrosis) may be increased in patients with ITP. The presence of grade $1 / 2$ reticulin was reported in the BM of up to $67 \%$ of patients with ITP. ${ }^{42}$ Reticulin is a normal component of the bone marrow and is commonly seen in patients with autoimmune disorders. This is reversible, quite unlike collagen fibrosis. Bone marrow examination was not a requirement of the randomized clinical trials. However of 117 ITP patients exposed to eltrombopag, 19 had bone marrow examinations (after a median of 13 months of various treatments) and seven showed fibrosis (5 reticulin and 2 collagen). ${ }^{15}$

A recent analysis of bone marrow biopsy reports in patients within EXTEND, REPEAT and RAISE clinical trials has been reported in abstract form. The reports of bone marrow biopsies performed at any time prior to eltrombopag exposure were reviewed. In the EXTEND study participants underwent bone marrow biopsy after one year on treatment and these reports were compared. There was no evidence of clinically relevant BM abnormalities or clinical findings typically associated with myelofibrosis in patients treated for up to 18 months with eltrombopag. Systematic longitudinal evaluation of bone marrows in EXTEND will provide meaningful data on the incidence of fibrosis during long-term treatment. ${ }^{43}$

\section{Increase in myeloblasts}

Myelodysplastic syndrome is a clonal disorder with a propensity to malignant transformation, usually to acute myeloid leukemia. Malignant hemopoietic cells are known to express TPO receptors and there is a theoretical risk that TPO growth factors may stimulate growth of malignant cells. However the first generation TPOs used in patients with AML did not show any increase in blast percentage or relapse rates. ${ }^{44}$ At present there has been no reported case of increased blasts following the use of eltrombopag. Equally there have been no reports of stimulation of solid tumors in any trials. In vitro effects of eltrombopag on proliferation, apoptosis, differentiation, colony formation, and malignant self-renewal of bone marrow mononuclear cells (BM-MNC) of patients with AML and MDS have however been assessed. Malignant BM-MNC did not show increased proliferation, or increased clonogenic capacity at concentrations of eltrombopag ranging from 0.1 to $30 \mu \mathrm{g} / \mathrm{mL}$ in vitro. Furthermore, eltrombopag was capable of 
increasing megakaryocytic differentiation and formation of normal megakaryocytic colonies from cultured cells of patients with AML and MDS. ${ }^{45}$

\section{Conclusion}

The successful use of eltrombopag in patients with chronic and often refractory ITP shows promise for this agent. At present eltrombopag has been licensed for use in ITP by the FDA and MHRA. It is available as $25-\mathrm{mg}, 50-\mathrm{mg}$, or 75-mg tablets administered orally once daily. Since food and divalent cations affect absorption, recipients should not eat food 2 hours before or after ingesting eltrombopag, and medications with divalent cations (eg, antacids or calcium) should not be taken. An important limitation of eltrombopag treatment is the time delay to platelet response (presumed secondary to the kinetics of stimulating platelet production) precluding its use in the management of acute bleeding when an urgent platelet increase is required.

Because the long term adverse events remain unknown, its current use may initially be restricted to the treatment of chronic ITP. We await the reporting of the long-term extension study in progress and postmarketing surveillance in ITP should provide further information. Given the unknown long-term safety of eltrombopag this re-opens the debate as to whom we should be treating with chronic ITP. Should we treat the absolute platelet count or just the patient who bleeds? It is unclear whether bleeding propensity correlates with platelet size, maturity or specific activity. Disease modifiers (eg, platelet granule content) have not been identified.

At present the authors would support the use of eltrombopag for "third line" treatment of patients with persistent or chronic ITP. Typically such patients would have been refractory to palliative treatments such as oral steroids and intravenous immunoglobulin/anti D and would have usually been treated with either splenectomy and/or rituximab or other immunosuppressive agents such as mycophenolate mofetil, ciclosporin, and danazol. Whether this agent should be used before splenectomy is less obvious, particularly if long-term use is required to maintain platelet levels. If long-term safety studies and postmarketing surveilence continue to show admirable safety profiles with continued use, then these agents are likely to become more popular and given the poor tolerability of steroids and the concern over the use of blood products, these agents may in the future become the preferred therapy for patients with ITP, especially if short-term use can allow patients to go in to remission.

\section{Disclosure}

The authors report no conflicts of interest.

\section{References}

1. Rodeghiero F, Stasi R, Gernsheimer T, et al. Standardization of terminology, definitions and outcome criteria in immune thrombocytopenic purpura of adults and children: report from an international working group. Blood. 2009;113(11):2386-2393.

2. McMillan R, Wang L, Tomer A, Nichol J, Pistillo J. Suppression of in vitro megakaryocyte production by antiplatelet autoantibodies from adult patients with chronic ITP. Blood. 2004;103(4):13641369.

3. Olsson B, Andersson PO, Jernas M, et al. T-cell-mediated cytotoxicity toward platelets in chronic idiopathic thrombocytopenic purpura. Nat Med. 2003;9(9):1123-1124.

4. Gernsheimer T, Stratton J, Ballem PJ, Slichter SJ. Mechanisms of response to treatment in autoimmune thrombocytopenic purpura. $N E n g l$ J Med. 1989;320(15):974-980.

5. Houwerzijl EJ, Blom NR, van der Want JJ, Vellenga E, de Wolf JT. Megakaryocytic dysfunction in myelodysplastic syndromes and idiopathic thrombocytopenic purpura is in part due to different forms of cell death. Leukemia. 2006;20(11):1937-1942.

6. Provan D, Stasi R, Newland AC, et al. International consensus report on the investigation and management of primary immune thrombocytopenia. Blood. 2010;115(2):168-186.

7. Guidelines for the investigation and management of idiopathic thrombocytopenic purpura in adults, children and in pregnancy. Br J Haematol. 2003;120(4):574-596.

8. Kimura H, Ishibashi T, Shikama Y, et al. Interleukin-1 beta (IL-1 beta) induces thrombocytosis in mice: possible implication of IL-6. Blood. 1990;76(12):2493-2500.

9. Bartley TD, Bogenberger J, Hunt P, et al. Identification and cloning of a megakaryocyte growth and development factor that is a ligand for the cytokine receptor Mpl. Cell. 1994;77(7):1117-1124.

10. Kuter DJ, Beeler DL, Rosenberg RD. The purification of megapoietin: a physiological regulator of megakaryocyte growth and platelet production. Proc Natl Acad Sci US A. 1994;91(23):11104-11108.

11. Metcalf D, Burgess AW, Johnson GR, et al. In vitro actions on hemopoietic cells of recombinant murine GM-CSF purified after production in Escherichia coli: comparison with purified native GM-CSF. J Cell Physiol. 1986;128(3):421-431.

12. Metcalf D, Begley CG, Johnson GR, Nicola NA, Lopez AF, Williamson DJ. Effects of purified bacterially synthesized murine multi-CSF (IL-3) on hematopoiesis in normal adult mice. Blood. 1986;68(1):46-57.

13. Carver-Moore K, Broxmeyer HE, Luoh SM, et al. Low levels of erythroid and myeloid progenitors in thrombopoietin-and c-mpl-deficient mice. Blood. 1996;88(3):803-808.

14. Gainsford T, Roberts AW, Kimura S, et al. Cytokine production and function in c-mpl-deficient mice: no physiologic role for interleukin-3 in residual megakaryocyte and platelet production. Blood. 1998;91(8):2745-2752.

15. Kuter DJ. Thrombopoietin and thrombopoietin mimetics in the treatment of thrombocytopenia. Annu Rev Med. 2009;60:193-206.

16. Rojnuckarin P, Drachman JG, Kaushansky K. Thrombopoietin-induced activation of the mitogen-activated protein kinase (MAPK) pathway in normal megakaryocytes: role in endomitosis. Blood. 1999;94(4):12731282.

17. Yang C, Li YC, Kuter DJ. The physiological response of thrombopoietin (c-Mpl ligand) to thrombocytopenia in the rat. Br J Haematol. 1999;105(2):478-485.

18. Stoffel R, Wiestner A, Skoda RC. Thrombopoietin in thrombocytopenic mice: evidence against regulation at the mRNA level and for a direct regulatory role of platelets. Blood. 1996;87(2):567-573.

19. Emmons RV, Reid DM, Cohen RL, et al. Human thrombopoietin levels are high when thrombocytopenia is due to megakaryocyte deficiency and low when due to increased platelet destruction. Blood. 1996;87(10):4068-4071.

20. Kosugi S, Kurata Y, Tomiyama Y, et al. Circulating thrombopoietin level in chronic immune thrombocytopenic purpura. Br J Haematol. 1996;93(3):704-706. 
21. Fanucchi M, Glaspy J, Crawford J, et al. Effects of polyethylene glycolconjugated recombinant human megakaryocyte growth and development factor on platelet counts after chemotherapy for lung cancer. N Engl J Med. 1997;336(6):404-409.

22. Basser RL, O'Flaherty E, Green M, et al. Development of pancytopenia with neutralizing antibodies to thrombopoietin after multicycle chemotherapy supported by megakaryocyte growth and development factor. Blood. 2002;99(7):2599-2602.

23. Komatsu N, Okamoto T, Yoshida T, et al. Pegylated recombinant human megakaryocyte growth and development factor (PEGrHuMGDF) increased platelet counts (PLT) in patients (pts) with aplastic anemia (AA) and myelodyplastic syndrome (MDS). Blood. 2000;96(11):1273

24. Vadhan-Raj S, Murray LJ, Bueso-Ramos C, et al. Stimulation of megakaryocyte and platelet production by a single dose of recombinant human thrombopoietin in patients with cancer. Ann Intern Med. 1997;126(9):673-681.

25. Vadhan-Raj S, Verschraegen CF, Bueso-Ramos C, et al. Recombinant human thrombopoietin attenuates carboplatin-induced severe thrombocytopenia and the need for platelet transfusions in patients with gynecologic cancer. Ann Intern Med. 2000;132(5):364-368.

26. Schiffer CA, Miller K, Larson RA, et al. A double-blind, placebo-controlled trial of pegylated recombinant human megakaryocyte growth and development factor as an adjunct to induction and consolidation therapy for patients with acute myeloid leukemia. Blood. 2000;95(8):2530-2535.

27. Basser RL, Rasko JE, Clarke K, et al. Randomized, blinded, placebocontrolled phase I trial of pegylated recombinant human megakaryocyte growth and development factor with filgrastim after dose-intensive chemotherapy in patients with advanced cancer. Blood. 1997;89(9): 3118-3128.

28. Basser RL, Underhill C, Davis I, et al. Enhancement of platelet recovery after myelosuppressive chemotherapy by recombinant human megakaryocyte growth and development factor in patients with advanced cancer. J Clin Oncol. 2000;18(15):2852-2861.

29. Somlo G, Sniecinski I, ter Veer A, et al. Recombinant human thrombopoietin in combination with granulocyte colony-stimulating factor enhances mobilization of peripheral blood progenitor cells, increases peripheral blood platelet concentration, and accelerates hematopoietic recovery following high-dose chemotherapy. Blood. 1999;93(9):2798-2806.

30. Nomura S, Dan K, Hotta T, Fujimura K, Ikeda Y. Effects of pegylated recombinant human megakaryocyte growth and development factor in patients with idiopathic thrombocytopenic purpura. Blood. 2002;100(2):728-730.

31. Li JZ, Yang C, Xia YP, et al. Thrombocytopenia caused by the development of antibodies to thrombopoietin. Blood. 2001;98(12):3241-3248.

32. Duffy KJ, Darcy MG, Delorme E, et al. Hydrazinonaphthalene and azonaphthalene thrombopoietin mimics are nonpeptidyl promoters of megakaryocytopoiesis. J Med Chem. 2001;44(22):3730-3745.

33. Duffy KJ, Price AT, Delorme E, et al. Identification of a pharmacophore for thrombopoietic activity of small, non-peptidyl molecules. 2. Rational design of naphtho 1,2-d imidazole thrombopoietin mimics. J Med Chem. 2002;45(17):3576-3578.
34. Erickson-Miller CL, DeLorme E, Tian SS, et al. Discovery and characterization of a selective, nonpeptidyl thrombopoietin receptor agonist. Exp Hematol. 2005;33(1):85-93.

35. Jenkins JM, Williams D, Deng YL, et al. Phase 1 clinical study of eltrombopag, an oral, nonpeptide thrombopoietin receptor agonist. Blood. 2007;109(11):4739-4741.

36. Bussel JB, Cheng G, Kovaleva L, et al. Long-Term Safety and Efficacy of Oral Eltrombopag for the Treatment of Subjects with Idiopathic Thrombocytopenic Purpura (ITP): Preliminary Data from the EXTEND Study. Blood (ASH Annual Meeting Abstracts). 2007;110(11):566.

37. Bussel JB, Provan D, Shamsi T, et al. Effect of eltrombopag on platelet counts and bleeding during treatment of chronic idiopathic thrombocytopenic purpura: a randomised, double-blind, placebo-controlled trial. Lancet. 2009;373(9664):641-648.

38. Weili Bao SH, Karpoff M, Bussel JB, Yazdanbakhsh K Improved Regulatory T Cell Activity in Patients with Chronic Immune Thrombocytopenia Purpura Treated with Thrombopoietic Agents 51st ASH annual meeting and exposition. Ernest N. Morial Convention Center, New Orleans, LA. 2009;684:279-282.

39. Grotzinger KM, Johnson FR, Hauber AB, Ozdemir S, Bala MV. Patients are willing to trade off efficacy, safety, and administration attributes of chronic Idiopathic Purpura (ITP) therapy: Results from a large North American discrete choice study. Blood (ASH Annual Meeting Abstracts). 2008;112(11):669.

40. Bussel JB, Marks KM. How effective is eltrombopag for the treatment of thrombocytopenia in patients with HCV infection? Nat Clin Pract Gastroenterol Hepatol. 2008;5(8):424-425.

41. Erhardt JA, Erickson-Miller CL, Aivado M, Abboud M, Pillarisetti K, Toomey JR. Comparative analyses of the small molecule thrombopoietin receptor agonist eltrombopag and thrombopoietin on in vitro platelet function. Exp Hematol. 2009;37(9):1030-1037.

42. Ghulam Mufti AB, Robert Hasserjian, Barbara Bain, David Kuter, Lyndah Dreiling, Janet Nichol. Bone marrow reticulin in patients with immune thrombocytopenic purpura. J Support Oncol. 2007;5(4):80-81.

43. Mansoor N, Saleh JBB, Oliver Meyer, Henrik Frederiksen, Diane Johnni, Manuel Aivado, Andres Brainsky. Results of bone marrow examinations in patients with chronic immune thrombocytopenic purpura treated with eltrombopag paper presented at: 51st ASH Annual MeetingDecember 5, 2009; Hall E (Ernest N. Morial Convention Center).

44. Kuter DJ, Begley CG. Recombinant human thrombopoietin: basic biology and evaluation of clinical studies. Blood. 2002;100(10):3457-3469.

45. Will B, Kawahara M, Luciano JP, et al. Effect of the non-peptide thrombopoietin receptor agonist eltrombopag on bone marrow cells from patients with acute myeloid leukemia and myelodysplastic syndrome. Blood. 2009;114(18):3899-3908.

46. Kuter DJ. New thrombopoietic growth factors. Blood. 2007;109(11): 4607-4616.

\section{Publish your work in this journal}

Drug Design, Development and Therapy is an international, peerreviewed open-access journal that spans the spectrum of drug design and development through to clinical applications. Clinical outcomes, patient safety, and programs for the development and effective, safe, and sustained use of medicines are a feature of the journal,

\section{Dovepress}

which has also been accepted for indexing on PubMed Central. The manuscript management system is completely online and includes a very quick and fair peer-review system, which is all easy to use. Visit http://www.dovepress.com/testimonials.php to read real quotes from published authors. 